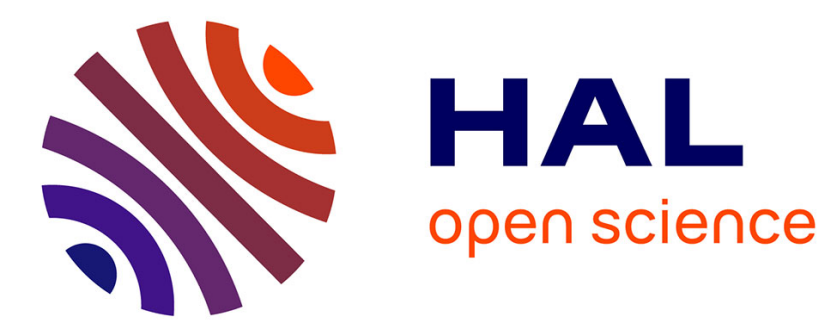

\title{
Associations islamiques et démocratie participative au Mali
}

Danielle Jonckers

\section{To cite this version:}

Danielle Jonckers. Associations islamiques et démocratie participative au Mali. A. Bozzo et P.J. Luizard (dir). Les sociétés civiles dans le monde musulman, La Découverte, pp.227-248, 2011, textes à l'appui/islam et société. halshs-00701896

\section{HAL Id: halshs-00701896 https://shs.hal.science/halshs-00701896}

Submitted on 28 May 2012

HAL is a multi-disciplinary open access archive for the deposit and dissemination of scientific research documents, whether they are published or not. The documents may come from teaching and research institutions in France or abroad, or from public or private research centers.
L'archive ouverte pluridisciplinaire HAL, est destinée au dépôt et à la diffusion de documents scientifiques de niveau recherche, publiés ou non, émanant des établissements d'enseignement et de recherche français ou étrangers, des laboratoires publics ou privés. 
in A. Bozzo et P.J. Luizard (dir) 2011 Les sociétés civiles dans le monde musulman, Paris,

La Découverte : 227-248.

\section{ASSOCIATIONS ISLAMIQUES ET DEMOCRATIE PARTICIPATIVE AU MALI}

\section{Danielle Jonckers}

La liberté d'expression et d'association s'exerce avec vitalité au Mali depuis l'insurrection de 1991 qui a mis fin à la dictature, instauré une démocratie parlementaire décentralisée et adopté une constitution conférant le droit à la désobéissance civile et réaffirmant la laïcité. Les citoyens interpellent les politiques, investissent l'espace public, s'expriment dans les médias et les radios libres, organisent des manifestations et des forums sociaux d'envergure internationale. Ils s'organisent en diverses associations qui concernent tous les domaines, y compris religieux. Le développement de centaines d'associations islamiques s'inscrit dans celui de milliers de pratiques associatives majoritairement non religieuses. Ces mouvements récents, librement constitués, s'appuient sur une habitude séculaire d'association maintenue en veilleuse sous les régimes colonial et dictatorial. D'usage tout aussi ancien et encore répandu en Afrique, les très codifiés rapports à plaisanteries ${ }^{1}$, qui concernent aussi bien des relations de parenté et d'alliance que de groupes plus vastes, se manifestent en tout liberté et dynamisent la pratique politique contemporaine. Ils autorisent des attitudes et des paroles d'affrontements, teintées d'humour qui permettent, à la fois de révéler des enjeux de pouvoir entre individus ou groupes, de négocier des alliances et de désamorcer les tensions. La profusion actuelle d'associations islamiques résulte de l'insatisfaction de l'exercice démocratique au sein des partis politiques, des syndicats ou d'ONG dont les liens avec les financements internationaux les mettent en décalage croissant avec les préoccupations des populations. Ces associations concernent l'ensemble du Mali et émanent de ce qu'on

\footnotetext{
${ }^{1}$ Pour une synthèse des recherches sur les relations plaisanteries et de nombreuses références bibliographiques voir le numéro 184 des Cahiers d'études africaines [CANUT, SMITH, 2006].
} 
appelle couramment la société civile. La complexité des rapports que cette notion recouvre sera interrogée à partir des réalités vécues et des initiatives distinctes des projets étrangers.

Nous envisagerons, en particulier, les adhésions individuelles aux associations islamiques, qui contribuent à porter les débats sur la place publique et à interpeller, grâce à la visibilité de l'islam, les autorités politiques et religieuses. Ces mobilisations traduisent des revendications identitaires ou sociales et les oppositions aux orientations de l'Etat prises sous la pression occidentale, notamment la réforme du code de la famille et les privatisations des entreprises publiques. Les dynamiques associatives seront analysées dans différents milieux de la capitale et de la région cotonnière du sud est du Mali où nous menons des recherches en anthropologie depuis $1971^{2}$. L'approche dans la longue durée et au regard de l'histoire écarte les découpages culturalistes ou ethnicistes. Elle permet d'éclairer les usages contemporains de la société civile et d'analyser les références à l'islam dans un pays où plusieurs religions et différents peuples ont toujours coexisté. Nous aborderons les questions de méthodes que soulèvent les dynamiques politiques et religieuses actuelles particulièrement rapides et paradoxales.

\section{NOTIONS DE SOCIETE CIVILE}

Les Maliens de toutes conditions manient avec nuances l'idée de société civile, vulgarisée depuis les années 1980 par les instances internationales, le FMI ou la Banque mondiale et par la classe politique. Ils distinguent clairement, parmi les nombreuses associations, celles d'origine locale des ONG étrangères. Mais aucun terme ne traduit la notion de société civile dans les langues locales, pas plus d'ailleurs que celles d'espace publique, de démocratie ou de laïcité, qui sont désignés en français quelle que soit la langue utilisée. Ces notions se rapportent effectivement à l'histoire politique occidentale et la plupart des chercheurs ne l'envisage à propos de l'Afrique qu'en référence à l'Occident et notamment au passé colonial. Il n'en demeure pas moins que des pratiques très anciennes, ainsi qu'actuelles, correspondent bien à ce qu'il est convenu de nommer société civile. Pour peu qu'on ne

\footnotetext{
${ }^{2}$ Elles ont donné lieu à des publications, notamment sur les associations paysannes (1994) et islamiques (2009), les effets du développement (1987, 1995b), les règles matrimoniales (1990), les conversions (1998), auxquelles on peut se rapporter pour l'analyse des transformations socioéconomiques, politiques ou religieuses.
} 
s'arrête pas à l'idée que les concepts théorisés à partir de l'Occident ne peuvent convenir aux pays non occidentaux. Les acteurs politiques et sociaux du monde entier invoquent la société civile, depuis une trentaine d'années, sans pour autant qu'on puisse lui attribuer un sens précis. Cette idée présuppose la possibilité d'associations distinctes de l'Etat et renvoie aux relations gouvernés-gouvernants en démocratie. Son utilisation permet de traiter des modes d'action politique populaires [Bayart, 1983], mais également de ceux qui émanent des institutions internationales.

Les ONG étrangères, représentant essentiellement la société civile pour les instances occidentales, sont pour la plupart liées aux réseaux du pouvoir politique et économique, au point que des chercheurs parlent d' "ONGisation » de l'Afrique [Lebeau et alii, 2003 ; Bayard 2004; Dozon, 2008]. Ces organisations reposent sur des logiques de courtage [Bierschenk et alii, 2000 ; Blundo, 1995], là où les autres comptent avant tout sur leurs adhérents. Les bailleurs extérieurs imposent une contrepartie. Ainsi, les ONG liées aux fonds de développement impliquent le recours aux entreprises et équipements étrangers et augmentent de ce fait la dette, tandis qu'une classe moyenne s'enrichit au détriment de la majorité [Georges et alii, 1994]. Sous couvert de bonne gouvernance, de développement durable ou de lutte contre la pauvreté, on prône la privatisation qui, certes, favorise la croissance $(4,5$ $\%$ en 2009), mais aussi son corollaire, l'augmentation de la paupérisation de la majorité de la population [Ziegler, 2005 ; Traoré, 1999]. Les secteurs vitaux de l'économie nationale (eau, électricité, transports, télécommunications, huilerie, compagnie cotonnière,...) ont ainsi été privatisés et bradés. Ce marché de l'aide dite humanitaire ou « coopération » s'inscrit dans la continuité de la «mission civilisatrice » de l'impérialisme colonial, ainsi que le soulignent l'anthropologue JeanPierre Olivier de Sardan [1997], le médecin cofondateur de Médecins sans Frontières, Rony Brauman [2009] et l'historienne, ancienne ministre malienne, Aminata Traoré [2002]. Nombre de Maliens critiquent les discours altruistes ou modernistes des ONG, alors qu'elles visent essentiellement à l'octroi de contrats et de revenus pour leurs agents. Ces courtiers du développement constituent effectivement de nouvelles élites disposant de plus de moyens que les pouvoirs locaux, la mairie ou l'hôpital, par exemple. 
L'appropriation populaire de la notion de société civile désigne des mouvements qui se démarquent des ONG ou des syndicats classiques. L'analyse de ces expériences, qu'elles soient en mesure ou non de déterminer des orientations politiques, ne relève pas seulement de l'activité de chercheurs pour lesquels la société civile africaine apparait souvent plus «comme une aspiration que comme une réalisation » [Miran, 2006, p. 398]. Elle est opérée par les intéressés qui font la distinction entre les associations qu'ils créent eux-mêmes et celles reconnues habituellement comme société civile, dont ils ne partagent pas nécessairement les projets, mais qu'ils acceptent néanmoins comme source de revenus, notamment les nombreuses ONG étrangères ou nationales. Quant aux syndicats et aux partis, ils correspondent souvent à des groupements d'intérêts économiques, indépendamment des convictions des élus. Leurs orientations politico-économiques sont alors susceptibles d'être déterminées par les instances internationales qui leur dictent les programmes susceptibles d'être financés.

De nombreuses manifestations et l'abstention massive aux élections (65\%) témoignent au Mali de l'indignation face à l'ingérence des institutions internationales et des pays occidentaux qui se posent en instituteurs du droit, de la démocratie, de la morale, de la santé ou de la famille, œuvrant à l'émancipation d'autres peuples qui seraient prisonniers de traditions ou de systèmes politiques archaïques. Les mouvements citoyens se multiplient et les associations islamiques, qui en font partie, représentent de nouvelles formes de sociabilité et d'action politique ou religieuses. Ces associations volontaristes diffèrent ici de celles du Sénégal ${ }^{3}$, créées par des confréries ou des marabouts aux visées politiques [Coulon, 1983; Seck, 2008, p. 65]. Elles permettent d'exprimer des positions politiques ou sociales, même si la pensée musulmane distingue, dans les faits, religion et politique, une idée difficile à faire admettre actuellement [Burgat, 2002, p. 201], et même si la Constitution laïque s'accompagne au Mali de lois strictes interdisant toute interférence entre les deux domaines ${ }^{4}$. Les motifs d'adhésion à ces associations varient selon les personnalités: moraliser la société, s'engager dans des luttes politiques ou faire valoir des revendications sociales et identitaires.

\footnotetext{
${ }^{3}$ Voir bibliographie indicative Samson F. (2005 : 353-366)

4 Voir les lois relatives aux associations 38, AN-RM, 5-8-2004 et à la laïcité 61-86, AN-RM, 21-71961, art 22 du code civil.
} 
Au regard des citoyens, les véritables représentants de la société civile n'entretiennent pas de liens avec le pouvoir ou un parti politique et se préoccupent de justice sociale. Porteurs de ces revendications, ils se distinguent des représentants des associations soutenues par l'étranger.

\section{Les représentants des villageois}

Lors d'une causerie, un modeste chef de village ne soutenant aucun parti déclare fièrement représenter la société civile, mais quand nous lui disons qu'il ne semble pas totalement dépourvu de pouvoir, même si celui-ci a diminué depuis la création des communes, il acquiesce et s'exclame «Donc, je ne suis pas la société civile!». Il représente cependant l'ensemble du village avec ses différentes composantes auprès du préfet de région. Ainsi, il accompagne chez ce dernier tout initiateur de projet concernant la collectivité, muni d'un document contresigné par les conseillers, par exemple, les représentants des différents cultes, pour une demande d'édification de sanctuaire, de mosquée ou d'église. Après enquête, la préfecture le charge de transmettre la décision à l'ensemble du village.

Les villageois précisent que le maire, lui, ne représente pas la société civile car il est membre d'un parti politique. Ils s'interrogent, par ailleurs, sur le parti Sadi (Solidarité Africaine pour la Démocratie et l'Indépendance) qui se fait leur porte-parole et dont ils aiment entendre les prises de position car, disent-ils, il les défend. Créé en 1996 pour rappeler les idéaux du mouvement insurrectionnel, Sadi représente l'opposition à l'Assemblée nationale où il se mobilise pour des revendications sociales, dont celles des paysans qui constituent la majorité de la population [Jonckers 2009, p.104-105]. Ils évoquent également la radio Kayira, "Aube nouvelle », en déclarant en chœur qu'elle dit la vérité, mais qu'elle n'a pas de pouvoir et en concluent qu'elle émane de la société civile. Le parti Sadi s'est constitué autour de la radio associative Kayira, mais celle-ci conserve sont indépendance. Elle n'hésite pas à donner la parole à ceux à qui on la dénie et à dénoncer les abus des détenteurs du pouvoir. Ces derniers, et ceux qui craignent les mouvements sociaux, la traitent de «radio des milles collines », nom de la radio qui appelait au génocide au Rwanda. Or, Kayira est proche de l'Association malienne des droits de l'Homme et 
fut une des premières radios à émettre en 1992 [Jonckers, ibid., p. 107109]. Ses stations représentent des pôles de vigilance démocratique dans toutes les régions du pays et constituent un réel contre- pouvoir. Ses auditeurs, qui la financent avec leurs faibles moyens, l'appellent «la Radio des Sans-voix » et celle-ci qualifie ses auditeurs d' "acteurs non étatiques de la société civile ».

Un débat s'engage à propos des religieux. Certains considèrent qu'ils ne peuvent représenter la société civile. Cependant, un consensus se dégage sur les associations religieuses, à condition que toutes les confessions soient prises en compte. Les non-musulmans signalent qu'ils apprécient pourtant le prêcheur musulman Haïdara ${ }^{5}$, ancien président de l'association Ançar Dine ${ }^{6}$ présente au village. Elle a débuté dans les années 1970 avec de jeunes cultivateurs musulmans de la région de Sikasso et atteint aujourd'hui une dimension internationale avec des associations aux Etats-Unis, en France, en Italie, en Suisse et dans une vingtaine de pays d'Afrique [Jonckers ibid., pp. 113-114]. Haïdara adresse régulièrement des reproches aux puissants, les faama (de fah fo, le détenteur de la force), politiciens, juges, hommes d'affaires ou forces de l'ordre précise-t-on en bamana [Jonckers, 1997]. Le bamana ou bambara, seconde langue, comprise dans les pays voisins, dispose de termes datant de l'Empire du Mali (XIII-XVIe siècles) et toujours d'usage pour distinguer différents aspects du pouvoir politique ou religieux, selon que l'on soit violent (faama), pacifique ou sacré (masa), intransigeant et équitable ( $k u n$ tigi, celui de la tête, le chef qui dirige) ou exemplaire (mogo tigi, celui des personnes, le guide que l'on suit). Haïdara est un mogo tigi qui s'oppose aux faama. Radio non confessionnelle, Kayira donna dès sa création la parole au jeune prêcheur interdit dans les mosquées pour ses critiques des politiques et religieux corrompus. Elle le met encore en onde sans pour autant devenir religieuse. Les radios (kuma so, maison de paroles) libres, associatives, commerciales, politiques ou religieuses, constituent le moyen d'information et de communication le plus adapté, les villages étant dépourvus d'électricité; elles sont trois cents à émettre depuis le changement de régime. Ces avis recueillis en milieu rural correspondent à ceux relevés en milieu citadin modeste ou chez tous ceux qui

\footnotetext{
${ }^{5}$ Il s'agit de Cherif Ousmane Madani Haïdara, couramment désigné par son nom ou plus familièrement par son prénom Madani ou Dani ; pour les références bibliographiques voir Jonckers, 2009 et Soares, 2004.

${ }^{6}$ Ainsi orthographié par l'association. ; en arabe Ansar al-din.
} 
s'intéressent aux dynamiques associatives, indépendamment de modèles préétablis.

\section{Représentants citadins}

Les villes où s'élèvent les sièges des associations donnent à voir les différents représentants de la société civile ainsi que de nombreuses manifestations où se croisent aussi bien des préoccupations sociales que politiques ou religieuses. Les porte-paroles des associations islamiques, au même titre que ceux de tout autre mouvement, s'expriment publiquement et peuvent être invités dans les débats politiques nationaux comme représentant la société civile à condition de ne pas s'opposer à la laïcité de l'Etat. Des lois strictes interdisent l'usage politique des institutions religieuses et l'usage religieux d'instances politiques [Jonckers, 2009, pp. 109-111]. Bien qu'elles en fassent partie, les associations islamiques fondées par les citoyens se distinguent du Haut conseil islamique institué par l'État ainsi que des associations financées par des Etats musulmans. Elles sont nettement majoritaires et relèvent du libre choix individuel. Elles peuvent être une alternative aux partis et syndicats ou se combiner avec la participation à ceux-ci et à d'autres associations.

Des représentants de la société civile d'orientations très diverses peuvent faire cause commune car il existe une réelle culture laïque. Ainsi, on remarque, aux côtes de l'archevêque de Bamako, le représentant du Haut conseil islamique, celui de l'Eglise protestante, ainsi que l'altermondialiste Aminata Dramane Traoré, le président de l'Association malienne des droits de l'Homme et les chefs de quartiers de Bamako, agir en tant que médiateurs d'un mouvement de travailleurs qui, en grève illimitée, occupent la Bourse du travail, le plan social proposé par la centrale syndicale, consécutif à la privatisation de leur entreprise, n'ayant pas abouti. Le Mouvement des Sans-Voix les soutient également et la radio Kayira fait écho à ces luttes, visibles aussi dans un film circulant en DVD au Mali, à l'étranger et sur internet [TV Bruits, SI CNT, Rebellyon TV]. Rappelons que, lors de l'insurrection populaire de 1991, le cinéaste Cheick Oumar Sissoko avait coordonné les associations opposées à la dictature pour former le Mouvement démocratique, auquel l'armée, qui s'insurgera par la suite contre le pouvoir, rendra hommage. En des styles différents, ces religieux ou ces militants dénoncent la privatisation des entreprises d'Etat votée par la majorité des députés et 
posent la question de la souveraineté d'un pays qui suit les injonctions de la Banque mondiale et du Fonds monétaire international. Ils organisent des conférences de presse, marches, meetings avec d'autres représentants de la société civile, intellectuels, écrivains, cinéastes, chanteurs et, bien entendu, le Forum des peuples et le Forum social mondial qui se tiennent régulièrement au Mali depuis 2002, rassemblant les mouvements sociaux du monde entier [Jonckers, ibid., pp. 105-106]. Le cinéaste mauritanien Abderrahmane Sissako, a magistralement mis en scène le procès des institutions financières internationales par la société civile, dans Bamako, sorti en 2006, où Aminata Traoré et des avocats maliens jouent leur propre rôle.

Le cadre politique et juridique du Mali permet ces initiatives qui s'écartent de la logique du capitalisme financier et convergent vers celles issues d'autres pays [Amin et alii, 2002 ; Houtart, 2001 ; Ziegler, 2002]. Lagrée [2004] qualifie de société civile internationale cette transnationalité des actions militantes, leur portée au-delà des frontières.

\section{Représentants au-delà des frontières}

Les forums sociaux diffusent les actions locales sur internet et, comme la majorité de la population malienne n'accède pas financièrement à internet ou est privée d'électricité, les radios associatives en réseau avec les cybermilitants altermondialistes transmettent les revendications locales dans le monde. On n'observe pas un tel relais informatique entre les associations islamiques, mais elles manifestent publiquement, et des adhérents assurent les contacts par téléphone mobile, tandis que des enregistrements sonores ou filmés circulent à travers le pays et au-delà des frontières.

La société civile englobe les Maliens travaillant à l'étranger. Un ministère les représente au Mali, ainsi que des associations, religieuses ou non, qui relaient leurs revendications, notamment des travailleurs sans-papiers en France et des expulsés. Celles-ci ont été entendues par le chef de l'Etat qui refuse de signer l'accord sur les flux migratoires exigé par la France et demande la régularisation des Maliens qui y vivent et y travaillent. Ces derniers contribuent à l'économie du Mali. Selon une étude de la Banque africaine de Développement (2005) [Keita, 2009, p.11], les Maliens de l'extérieur, représentant un tiers de la population, transfèreraient 458 millions d'euros par an vers le Mali, l'équivalent de 
$79 \%$ de l'aide publique au développement qui, elle, accroît la dette, et $12 \%$ du PIB. A ces transferts officiels s'ajoutent d'importantes sommes remises directement aux parents des intéressés par des voyageurs. Les Maliens résidant en France fourniraient ainsi 183 millions d'euros, l'apport individuel s'élevant à 1402 euros par an. Par ailleurs, des adhérents d'Ançar Dine établis à l'étranger cotisent pour inviter le prêcheur Haïdara, notamment dans leur foyer parisien et réaliser un DVD ou se rendre au Mali pour des célébrations telles le Maouloud. Les uns analysent leur situation en des termes comparables à ceux des altermondialistes, tandis que d'autres privilégient le mode religieux et se réfèrent aux premiers temps de l'islam, mais, dans les deux cas, face aux conséquences du libéralisme économique. Car, au-delà de leur idéologie religieuse, c'est bien le respect des droits humains qu'ils font valoir, ainsi que le souligne Burgat [2005]. D'autres encore s'accommodant de l'économie actuelle et investissent dans le foncier urbain [Schmitz, 2008, p. 12].

Une autre société civile est aussi souvent évoquée, particulièrement éloignée des réalités sociales, mais qui bénéficie d'une reconnaissance des puissances occidentales et de financements qui se comptent en millions ou milliards de francs CFA. Ces organisations sont inévitablement en relation étroite avec le pouvoir [Lagrée, 2004]. Rassemblant des représentants d'ONG, d'organisations paysannes, d'associations de femmes et de syndicats, de nombreuses consultations et commissions, devant des consultants étrangers, débouchent sur des rapports, qualifiés de Plan de Stratégie nationale, qui encadrent la politique budgétaire du gouvernement malien selon les exigences de la Banque mondiale. Les ONG formatent également leurs projets en ce sens pour obtenir des financements. Il existe ainsi un Conseil national de la société civile chargé d'inciter la dite société civile, essentiellement les ONG, à participer aux politiques de développement dans le cadre du CSCRP (Cadre stratégique de croissance et de réduction de la pauvreté). Dans le même but, un Forum des Organisations de la Société Civile a été officiellement inauguré en avril 2009. Il dispose d'un portail sur internet, qui met régulièrement à jour les activités du mouvement associatif. A titre indicatif, notons, sur leur site, la brève du 10 mars 2009 annonçant le lancement d'un fonds d'investissement pour les entrepreneurs, faisant l'éloge d'un homme d'affaire malien, celui-là même qualifié de faama par la société civile d'en bas [Houtart, 1998]! 
L'Etat malien entend structurer la société civile en créant l'espace d'interpellation démocratique qui informe les opinions nationales et internationales sur les droits de l'homme au Mali. Mais, rompant avec le droit en vigueur, il réprime en 2008 les manifestations contre la privatisation de l'eau, la "marche des démunis », les activistes "SansVoix » et Kayira. Dans la volonté de se conformer, comme les pays voisins, aux normes occidentales, il fonde en 2002 le Haut conseil islamique, qui se veut représenter l'ensemble des musulmans, soit 80 à $90 \%$ de la population, dans la mesure où il compte des représentants des associations islamiques et de tous les ordres religieux. En politique extérieure, il donne du Mali une image musulmane exempte de radicalisme aux pays arabes et occidentaux, puissants ou partenaires financiers, prompts à voir un danger islamiste dans la prolifération des associations islamiques. En politique intérieure, il maintient sous contrôle les tendances d'opposition des divers courants, qualifiés, selon les points de vue, de mystique, réformiste ou fondamentaliste, qui animent les ordres de l'islam sunnite (Tijaniyya, Quadiriya, Hamalliyya, Wahhabiyya et Salafiya) ${ }^{7}$. Celui-ci, dépourvu de structure cléricale, a des religieux indépendants qui n'entretenaient que des rapports indirects avec le pouvoir. Bien que des loges existent, il n'y a pas non plus de réseaux confrériques de personnalités musulmanes étroitement liées au politique, comme au Sénégal et en Mauritanie [Ould Cheikh, 2004, Robinson, 2004, p. 26], où ce système s'est développé sous la colonisation française. Le Haut conseil islamique pourrait entraîner des usages politiques du religieux, même s'il ne s'agit pas pour l'Etat d'islamiser les institutions et si les partis religieux sont interdits. Mais il n'est pas impossible que certains de ses membres ne tirent avantage de la légitimité que leur confèrent les négociations avec le pouvoir et se lancent dans des projets politiques personnels. Le Haut conseil représente la société civile pour l'Etat mais les associations qu'il regroupe ont conservé leur autonomie, leurs orientations propres, comme en Côte d'Ivoire [Miran, 2006, p. 410] et, pour certaines, maintiennent une force d'opposition, ce qui n'est pas le cas dans d'autres pays à régime autoritaire [Burgat, 2002, p. 264]. Haïdara, membre du Haut

\footnotetext{
${ }^{7}$ L'ordre Quadiriya est présent depuis le XII ${ }^{\mathrm{e}}$ siècle, le Tijaniyya le XVIII et les plus récents le $\mathrm{XX}^{\mathrm{e}}$, le Wahhabiyya, depuis les années 1940 et le mouvement Salafi minoritaire, à partir de 1970. A propos des ordres et courants musulmans et pour les références bibliographiques voir Jonckers [2009, p. 116$117,127-130]$.
} 
conseil, représente Ançar Dine, l'association la plus populaire qui rassemble des adeptes des différents ordres religieux, et il n'a rien perdu de son franc parler. La société civile d'en bas le reconnait comme porteparole quand il se lance publiquement dans de violents réquisitoires contre les détenteurs du pouvoir politique. Sa parole, légitimée au nom de l'islam, donne du poids à des revendications sociales qui n'ont rien de religieux. De ce fait, certains le disent «islamiste », tandis que d'autres, au contraire, l'honorent comme un saint soufi [Soares, 2004, p. 219]. Dans les années 1990, les adhérents d'Ançar Dine critiquaient, comme le font les Salafis ${ }^{8}$ et les Wahhabites/Sunnites ${ }^{9}$, les pratiques soufies, ainsi que le clientélisme de religieux qui commercialisent leurs services ou la visite à la tombe des ancêtres [Soares, 1996 ; Schulz, 2006]. Mais il y a une tendance actuelle, encouragée par l'État, d'adopter le point de vue occidental qui valorise une forme de culture soufie comme rempart contre l'islamisme ${ }^{10}$ [Burgat, ibid., pp.100-106].

\section{ISLAM ET ESPACE PUBLIC}

La légitimité reconnue des actions de revendications sociales et d'oppositions politiques ou religieuses, distingue le Mali de la plupart des pays musulmans à pouvoir fort. L'islam s'y pratique depuis des siècles, mais ni l'Etat ni les institutions ne sont islamiques et aucune valeur juridique n'est reconnue au droit musulman malékite. L'exemple du Mali est intéressant d'un point de vue comparatif dans la mesure ou la laïcité est un fait établi et n'était pas l'objet d'enjeux politiques ou idéologiques. La majorité de la population ne met pas la laïcité en question en tant que telle, mais s'insurge contre la mauvaise qualité des institutions, le manque de civisme des autorités ou les orientations du gouvernement prises sous la pression internationale. Des associations islamiques relaient ces critiques et pallient les manquements des institutions laïques dans les domaines de la santé et de l'enseignement. Elles contribuent certes à la visibilité de l'islam, correspondant à une volonté d'affirmation identitaire des musulmans, mais elle est populaire car elle symbolise également une modernité en rupture avec des modèles culturels africains ou occidentaux insatisfaisants pour la plupart des

\footnotetext{
${ }^{8}$ Les Salafis, comme la majorité des musulmans, se démarquent des Salafistes armés minoritaires dans le Nord du Mali et les pays voisins et privilégient le courant piétiste salafi,

${ }^{9}$ Les adeptes réfutant la désignation Wahhabites, nous optons pour la double appellation Wahhabites/Sunnites adoptée par Brenner (1993a : 60) qui traite de l'émergence de cet ordre au Mali (60-67). Voir également les références bibliographiques qu'il mentionne (60, note 4).

${ }^{10}$ Pour une approche nuancée de l'islamisme voir BURGAT, 2002 (1995), 2005.
} 
Maliens.

Les musulmans ne forment pas un ensemble social ou religieux homogène et leurs orientations vont de la laïcité au fondamentalisme religieux. Ils n'ont pas une spécificité à se regrouper : leurs associations sont comme les autres, librement constituées et légalement enregistrées. Ils participent également aux associations non religieuses majoritaires. On compte deux cents associations islamiques parmi les 18720 associations déclarées en 2008 et enregistrées au ministère de l'Intérieur, auxquelles s'ajoutent des dizaines de milliers d'organisations non enregistrées. La plupart revendiquent le respect de principes partagés par la majorité des citoyens, indépendamment de leur appartenance religieuse. L'activisme de certains s'articule à l'amplification des mouvements sociaux. Les femmes et les jeunes mobilisent en nombre leurs associations ainsi l'Union des Jeunes Musulmans du Mali regroupe plus d'une centaine d'associations de la jeunesse musulmane. Il y a, au Mali, une conception de l'espace public, combinant les domaines religieux, sociaux ou politiques, qui le distingue du modèle d'Habermas, où la religion trouve difficilement sa place, ainsi que le note Soares [2004, p. 207].

La démocratie a permis l'exercice des droits et la liberté de paroles ou de pratiques religieuses autrefois cantonnées au domaine privé. Depuis une vingtaine d'années, les musulmans investissent l'espace public, lieu d'interpellation autonome par rapport à l'Etat et la sphère privée. Ce qui a amené des chercheurs à parler de «société civile islamique » pour désigner l'entrée des musulmans dans l'arène politique en Côte d'Ivoire, en tant que contre-pouvoir [Miran, ibid., pp. 397-441] ou d'" espaces publics religieux ». Or, c'est en référence à la laïcité et à la pluralité religieuse garanties par la Constitution que les musulmans expriment aujourd'hui au Mali les courants les plus divers. Cela signifie l'adhésion à des valeurs citoyennes, le respect d'un espace sociétal commun où les différences religieuses peuvent s'exprimer publiquement. Haïdara rappelle aujourd'hui ces principes qui permettent à chacun de pratiquer librement sa religion : "Si vous êtes musulman, on vous laisse faire vos prières. Si vous êtes chrétien, on vous laisse avec votre foi. Si vous êtes féticheur, on vous laisse adorer vos fétiches. » Ses déclarations publiques, largement diffusées [Schulz, 2006], sont réécoutées des années durant. Les moins tolérantes, ont la préférence de conservateurs 
qui les programment sur les radios qui toutes, quelle que soit leur orientation politique, accordent, au nom de la liberté d'expression, un temps d'antenne aux religieux dont les messages contrastent parfois avec la ligne éditoriale de la station.

Les mouvements islamiques ne sont généralement pas prosélytes; ils s'adressent aux musulmans et coexistent avec les autres formes d'expressions religieuses, certes minoritaires, mais également visibles dans l'espace public, comme celles des cultes bamana, chrétiens ou protestants. Le prosélytisme de ces derniers a amené des musulmans à manifester publiquement leur piété, à sortir de la marginalité sociale et de l'ignorance doctrinale. Les fidèles vont à la mosquée le vendredi ou prient dans les rues et sur leurs lieux de travail ou d'étude. Ils se rendent au stade omnisport pour écouter un prêcheur populaire, mais ce terrain

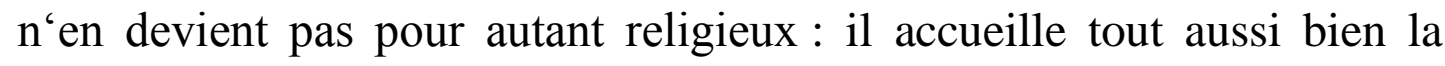
foule pour de célèbres chasseurs, magiciens, musiciens, chanteurs ou des sportifs, bien entendu, dont des équipes féminines réputées. Les mosquées étaient rares et peu fréquentées jusque dans les années 1970. Les musulmans manifestaient peu leurs options religieuses collectivement, à l'exception de cérémonies ponctuelles de baptême ou de décès dans certains milieux. Depuis des siècles, des lieux de pèlerinages existaient dans le nord et l'ouest du pays et des musulmans se rendaient en caravanes à la Mecque mais la majorité n'y allait pas. Aujourd'hui, il y a milliers de pèlerins. De nombreuses agences de voyages maliennes proposent les pèlerinages, et affrètent spécialement des avions. Elles font partie de la commission nationale d'organisation du pèlerinage aux lieux saints. L'Etat malien contrôle les filières gouvernementales et privées, en charge du transport et de l'encadrement des fidèles.

La volonté de visibilité des musulmans s'amplifie en opposition à l'ingérence occidentale qui s'affiche de la capitale aux villages sillonnés par des véhicules aux sigles des ONG et des institutions internationales. Les projets de celles-ci font la une des médias, mais actuellement les réalisations des associations islamiques (écoles, hôpitaux, évènements, etc.) sont également mises en évidence et Haidara donne de nombreuses conférences de presse. Dans les villes, de grandes mosquées de style oriental, financées par des pays musulmans, ont vu le jour, contrastant avec la plupart des autres, également récentes, dues aux apports des 
migrants en Europe et aux Etats-Unis. Ces dernières édifiées aussi dans les villages affirment la référence à l'islam qui leur permet de s'inscrire dans le monde et qui symbolise la modernité, la dignité et la prospérité (Jonckers 1998) malgré les difficultés locales et de l'exil.

Les femmes contribuent activement à la visibilité de l'islam par des tenues couvrantes dont le port ne repose sur aucune habitude locale. Le message véhiculé par le hidjab, voire le niqab, marque une rupture avec des cultures où les femmes ont presque toujours été tenues à l'écart du savoir religieux. Le voile n'est pas imposé, mais adopté par les femmes, qui prennent ainsi leurs distances par rapport à la majorité musulmane peu rigoureuse. Il reflète un état d'esprit qui transcende la question du port de signes religieux. Il correspond à un idéal d'autonomie et d'émancipation. Des femmes, qui occupent des responsabilités, s'en drapent et des villageoises, même non musulmanes, se coiffent de foulards quand elles se rendent à des fêtes ou au congrès d'un parti politique. Précisons que les femmes sont présentes dans les partis, les syndicats, les ONG et au plus haut niveau du pouvoir: ministre, présidente de la Cour suprême, préfète ou membre du Haut conseil du culte musulman. Dans la foule des citadins, il n'est pas rare de voir des musulmanes en tenues saoudienne noire (jilbab) et même parfois afghane (burka). Par ces vêtements, importés de Chine, peu coûteux sur le marché, elles veulent ainsi donner l'image de femmes instruites en religion et ayant les moyens d'avoir accompli le pèlerinage. Nombre de femmes se consacrent désormais aux études religieuses, assument des enseignements avancés en religion, se chargent de responsabilités liturgiques ou rituelles [Tamari, 2010]. Les innovations vestimentaires symbolisent aussi l'opposition à l'Occident et font référence à la situation des musulmans dans le monde, particulièrement en Palestine, et à l'attitude des Etats-Unis ou de la France. Ainsi des hommes se couvrent la tête ou les épaules du khéfié ou ghotra, foulard rouge du golfe ou noire des Palestiniens et certains portent la tenue blanche des saoudiens dont la sobriété se démarque de l'habituel boubou de style musulman.

Depuis 2003, des rassemblements célébrant la naissance du Prophète, le Maouloud, ont lieu dans tout le pays, alors que, par le passé, ce n'était pas une fête populaire. Retransmis par la télévision, ils reflètent 
également des enjeux politiques à la portée internationale. On interprète la présence ou l'absence du chef de l'État ou de membres du gouvernement et de personnalités religieuses maliennes aux côtés de diplomates occidentaux et d'autorités libyennes ou iraniennes. C'est aussi à cette occasion de bénédictions et de longues heures de prières, qu'Haïdara, très médiatisé, devant plus de 20000 personnes rassemblées dans le stade de Bamako, critique, pendant le prêche, les autorités religieuses et politiques du pays, la délinquance financière et la justice qui piétine le droit des pauvres.

\section{FAITS ASSOCIATIFS ET HISTOIRE}

Les associations s'inscrivent dans des logiques économiques, politiques ou religieuses différentes selon les époques. Leurs formes actuelles ne découlent pas des anciennes. Mais il existe une habitude culturelle d'articulation de l'individuel (jon kani) et du collectif (foroba), du local et du global [Jonckers, 1994]. Les sociétés d'Afrique de l'Ouest ont très tôt ${ }^{11}$ entretenu et développé des rapports à l'Etat, que leur mode politique local admette ou non la centralisation du pouvoir. Ces rapports reposaient sur des relations d'alliances et d'oppositions modulables aux niveaux local, régional et de l'Etat. Autant de liens qui pouvaient s'articuler à ceux des cultes, en réseaux étendus, qui pouvaient donc avoir une portée politique [Amselle, 1990 ; Jonckers, 1995a; Saul, Royer, 2001]. Ainsi que le souligne Dahou [2005], l'anthropologie du politique a longtemps occulté ces interactions par des théories opposant sociétés à Etat et sociétés segmentaires. Elle a également éludé l'importance des délibérations publiques et des recherches de consensus. Les associations locales selon l'âge, le sexe, les fonctions, la religion, etc. constituaient des espaces de délibération dans le vaste Empire du Mali (XIIIe-XVIe siècles), qui s'étendait du Sénégal au Niger actuel, et était présent sur la scène internationale. Ces associations n'ont pas disparu au Mali. Elles ont perduré malgré la violence des Etats guerriers (XVIe-XVIIIe siècles) qui se situaient dans l'économie esclavagistes mondiale. La plupart des Etats ont laissé leur autonomie politique et religieuse aux royaumes, aux confédérations et aux villages. Certains chefs d'État ouest africains furent musulmans, d'autres non, mais les institutions et l'État n'ont été islamisées qu'à de rares exceptions. Des

\footnotetext{
${ }^{11}$ Les écrits arabes situent au IVe siècle après J.C. la fondation du Ghana qui s'étend à son apogée (IXXI) du Sénégal au Mali actuel et est connu en Europe et en Arabie pour son commerce de l'or et du sel.
} 
conseillers représentaient les diverses religions et les différents droits pratiqués. La colonisation française a institutionnalisé le recours à la force et privilégié l'emploi d'agents musulmans dans l'administration. C'est elle qui a établi des écoles et des juridictions musulmanes, même là où l'islam était minoritaire [Jonckers, 2006]. Les conversions à l'islam ont augmenté [Jonckers, 1998] et un réseau de clientèle de musulmans liés au pouvoir en résulta. Ce réseau se maintient jusqu'à présent, malgré quelques années d'éclipse durant la lutte pour l'indépendance et le régime socialiste qui suivit.

Benjamin Soares [2004, p. 214-215] évoque les associations musulmanes qui se forment dans les villes ouest africaines dès les années 1930. Elles organisent des conférences sur la morale musulmane ou les pèlerinages à la Mecque financés par les autorités et remercient la France pour sa «mission civilisatrice ». Dans les années 1950, des intellectuels musulmans brassent à la fois des idées politiques anticolonialistes et religieuses réformistes de l'islam. Des prêcheurs réformistes critiquent les autorités françaises et musulmanes. Ils sont réprimés, emprisonnés et leurs écoles fermées. Des intellectuels réformistes constituent l'Union culturelle musulmane avec le Sénégal et la Guinée. Ils prônent un islam rénové, l'accès à l'éducation. Certains publient des pamphlets anticolonialistes en français et en arabe, dénonçant le trio «capitaliste, marabout (religieux musulman), colonialiste», qui exploitent le peuple en Afrique Occidentale française. Ils critiquent les clercs musulmans pour leurs pratiques non islamiques. On retrouve des préoccupations identiques dans les associations islamiques actuelles [Soares, ibid.].

Des associations ancrées dans l'histoire de l'Empire du Mali se sont perpétuées en adéquation avec les réalités de chaque époque et demeurent très actives aujourd'hui ${ }^{12}$. C'est le cas des associations de griots $^{13}$, qui sont des musiciens historiens, et de chasseurs, à la fois thérapeutes, devins et magiciens. Présentes du village à la capitale, leur portée dépasse leurs aspects parfois folkloriques. Elles se déploient audelà des frontières tracées par la colonisation, au Burkina Faso et en Côte d'Ivoire. Elles rehaussent de leur présence les cérémonies

\footnotetext{
${ }^{12}$ Pour un aperçu des références sur ce socle historique, voir Ba Konaré [2009, p. 306-320 et p. 325338].

${ }^{13}$ Dans un de ses nombreux ouvrages, l'écrivain Francis Bebey (2008), qui fut aussi musicien et chanteur, traite avec humour des relations au griot, en politique d'un Etat contemporain fictif.
} 
officielles et les meetings politiques par des danses et des chants qui consacrent des valeurs séculaires et louent le fondateur de l'Empire du Mali, Soundjata Keita, ainsi que des personnalités contemporaines. Elles peuvent, dans certaines circonstances, jouer un rôle militaire. Lors des conflits frontaliers avec le Burkina (en 1976-77 et en 1985-86), les chasseurs ont ainsi proposé leurs services au gouvernement malien. Egalement héritées du passé et actualisées, les relations à plaisanteries sont aussi à l'œuvre dans l'espace public de plusieurs pays. L'année 2003 a été décrétée, au Mali, année de promotion de ces relations nommées senankuya, en bamana, et le Burkina ménage des plages horaires dans les programmes officiels, qui leur sont consacrées [Canut, Smith, 2006, p. 23]. Les relations à plaisanteries permettent à la fois la subversion et le maintient de la paix dans les rapports politiques, religieux ou de parenté et d'alliance et articulent les relations aux autres dans les enjeux de pouvoir [Amselle 1996]. Elles s'utilisent à diverses fins, allant de l'obtention de l'hospitalité au soutien électoral, en passant par des attentions ou des critiques voilées, voire à avoir la vie sauve, selon les stratégies des acteurs et le contexte. Elles ne sont pas figées, ce qui permet des recompositions d'alliance en politique, qualifiées de «senankuyamania» par Denis Douyon [2006]. Les Maliens de tous les milieux les utilisent à tous les niveaux, qu'ils soient au pays ou à l'étranger, chef de l'Etat, cultivateur, ambassadeur, épouse, politicien, prêcheur, etc.. Chacun, selon son style, peut y avoir plus ou moins recours. Les relations à plaisanteries font partie des modes d'expression actuels de la société civile et du monde politique au Mali comme ailleurs dans plusieurs pays d'Afrique.

Le chef de l'Etat, les musiciens de jazz, rock ou reggae et les médias se font l'écho des ressources du passé propices à la démocratie ou à la décentralisation [Jonckers 2009, p. 122]. Ce sentiment est largement partagé. On s'étonne que des chercheurs puissent les qualifier d'inventions néo-fonctionnalistes imputables aux financements des acteurs de développement extérieurs [Canut, op.cit., p. 82]. Les senankuya étaient en usage avant le règne des ONG. Certes, elles sont plus présentes dans l'espace public depuis la démocratie qui leur permet de s'exprimer encore plus largement, mais elles ont perduré sous tous les régimes et dans le privé. Des réseaux d'alliances et de clientèle anciens persistent également à tous les niveaux de la société et peuvent interférer dans les enjeux politiques ou économiques. Il y a une continuité 
historique entre le pouvoir actuel et les régimes antérieurs. Mais il n'est pas interdit de se départir de ces rapports interpersonnels en créant une association ou un parti et en s'exprimant librement dans les radios privées qui contrebalancent les médias monopolisés par l'État.

\section{OPPOSITION A LA REFORME DU CODE DE LA FAMILLE.}

Les résistances de la population à la réforme du code de la famille rappellent qu'il y a lieu de distinguer l'action publique mise en œuvre par les politiques et les organisations internationales de celle exercée par les citoyens. Elles mettent en évidence la complexité des enjeux de pouvoir dans les rapports de genre ainsi qu'en politique au niveau national et international. Les observateurs occidentaux attribuent ces résistances aux associations islamiques qui manifestent massivement leur opposition à la réforme. Ce point de vue est partagé par des Maliens, agents d'ONG, politiques ou universitaires, dont la carrière, modelée par la culture occidentale, les éloigne des réalités sociales. Mais d'autres, également critiques envers les religieux, reconnaissent l'impopularité de la réforme. Il s'avère que ces associations islamiques sont actuellement les porte-paroles de la population en majorité musulmane. Indépendantes des partis, des ordres religieux ou de financements extérieurs, elles pourraient être des voies originales pour faire pression sur les milieux politiques et économiques.

Leur étude impose une démarche réflexive et soulève des questions de méthode (Jonckers 2009, p.101-102). Nous n'avons pas présupposé leur importance, nous les avons découvertes au fur et à mesure en partageant la vie quotidienne des gens, leurs implications dans des institutions et manifestations politiques ou religieuses locales mais d'importance nationale. Nous avons été à l'écoute des ruraux et nous les avons accompagnés dans leurs diverses associations, aux cours de religion, aux prêches, aux cultes des différentes religions mais également aux conseils villageois et communaux, aux congrès de partis politique ainsi que dans les studios de radios. En ville, à Koutiala, nous avons écouté les propos du procureur, du juge, de députés, de journalistes de radio ou de membres influentes d'associations de femmes musulmanes et à Bamako, nous avons suivi ceux de femmes ministres ainsi que ceux de représentants d'associations. La presse, les rapports et les archives ont 
été dépouillées. Des analyses comparatives ont été menées auprès d'équipes de recherche travaillant dans différents milieux et, pour saisir les nuances des différents courants islamiques, nous avons suivi le premier séminaire sur le salafisme en 2007 et 2008 à l'Institut d'études de l'islam et des sociétés du monde musulman de l'EHESS à Paris. Nos observations, depuis 1992, se sont déroulées lors de cinq séjours de plusieurs mois dans une commune rurale, dans la ville de Koutiala et la capitale. Elles se situent dans la continuité d'enquêtes antérieures. L'analyse des associations islamiques dans le long terme s'impose pour évaluer leur importance et percevoir la complexité des trajectoires politiques et sociales des adhérents.

Les femmes s'impliquent particulièrement, mais tandis que les associations, créées et financées par une majorité d'entre elles, font référence à l'islam pour bloquer la réforme d'autres organisations, auxquelles des femmes participent également, y sont favorables et disposent de crédits importants. Les femmes des deux bords revendiquent le droit au choix individuel ou à la modernité [Schulz, 2003] et exercent une pression considérable sur les pouvoirs publics.

Le code actuel repose sur le droit positif. La réforme vise à confirmer l'égalité entre les femmes et les hommes, déjà mentionnée dans la constitution, les droits civils, économiques et sociaux, effectifs pour les femmes, et les traités internationaux ratifiés par l'Etat. Le projet de réforme entamé dans les années 90 prévoit également la pénalisation de l'excision et il est soutenu par les associations des droits des femmes, nationales et étrangères. Ces appuis extérieurs provoquent une vive opposition, car ils symbolisent l'ingérence de l'Occident. L'excision touche $90 \%$ des femmes, et, malgré les campagnes très médiatisées, elles refusent l'ingérence étrangère ou masculine dans cette pratique identitaire généralisée, toutes religions confondues. En 1996, le Comité national d'Action pour l'abandon des pratiques néfastes à la santé de la femme et de l'enfant est créé et en 1999 le ministère de la santé interdit l'excision dans les structures sanitaires et aux agents de santé. Mais la pratique se poursuit ailleurs. En 2006, la Ligue des droits des femmes interpelle la ministre de la Promotion de la femme qui rétorque qu'on n'impose pas une loi d'interdiction sous la pression internationale, même si son vote est prévu. En 2007, ministère et ONG participent aux journées d'études sur l'excision organisées par les leaders religieux 
musulmans avec le Haut conseil islamique et des scientifiques. Les religieux adoptent la «Déclaration de Ségou » qui rappelle que cette coutume n'est pas une obligation absolue de l'islam. On en fait état en France sur le site du Service des droits des femmes et de l'égalité. Mais au Mali, l'avis des religieux ne semblent pas avoir plus d'impact que le projet de loi pour abolir l'excision. Les actions gouvernementales et occidentales se poursuivent, mais la pratique persiste. Radio Kayira, qui diffuse les campagnes d'abolition, est à chaque fois très critiquée par des auditeurs. Une vingtaine de villages seulement sur 13000 et quelques exciseuses déclarent arrêter l'excision. En 2010, Haïdara, interpellé par une ONG belge lors de la célébration du Maouloud, rappellera que l'excision n'est pas une prescription de l'islam. Des Musulmanes et des religieux qui y sont favorables, précisent que le prophète, découvrant cette coutume, ne l'a pas interdite.

Depuis 2002, la réforme se concentre sur le code de la famille et ne mentionne plus explicitement la pénalisation de l'excision. Notons cependant que sa condamnation tombe déjà sous l'article 1 de la constitution qui garantit l'intégrité de la personne, de l'article 5 qui interdit d'y porter atteinte et que l'excision est juridiquement assimilée à coups et blessures volontaires (art $207 \mathrm{CP}$ ). Mais, à notre connaissance, aucune plainte n'a été portée devant les tribunaux.

Certaines associations islamiques sont proches du pouvoir politique ou des religieux tandis que d'autres s'y opposent. Mais lors des élections de 2002, elles ont remis un mémorandum collectif de défense des valeurs musulmanes aux différents candidats à la présidence de la république. Elles demandent l'intégration du droit musulman dans le code de la famille au même titre que le droit positif en vigueur (MAGASSA, 2005 : 21). Parmi elles, la puissante Union nationale des associations des femmes musulmanes du Mali (UNAFEM) qui regroupe une centaine d'associations féminines. Elle représente un tel potentiel d'électeurs qu'elle a une portée politique et aux élections présidentielles de 2002, un candidat reprend ses arguments religieux mais il ne sera pas élu.

En 2009, l'Assemblée nationale ratifie à l'unanimité la réforme. La réaction est immédiate et le Haut conseil islamique lui-même appelle à manifester. Les protestations fusent dans tout le pays. Les mobilisations ont une telle ampleur que le Président de la République renvoie le 
nouveau code aux députés en deuxième lecture. Haïdara salue sa sagesse et rappelle que le Mali n'est pas un Etat islamique, mais un pays laïc qui appartient autant aux chrétiens qu'à d'autres composantes. Il se démarque ainsi de personnalités d'obédience wahhabite-sunnite au Haut Conseil islamique.

Une commission est constituée par les représentants des familles fondatrices de Bamako, du Haut conseil islamique, des Eglises catholique et protestante, du Conseil national de la société civile et du barreau. Il semble que les questions litigieuses seront prises en compte. Elles ne sont pas, à proprement parler, religieuses, mais plutôt économiques et sociales. Rappelons que, tous milieux et toutes religions confondus, la majorité des mariages ne se faisait pas devant l'officier de l'état civil jusqu'à la création de communes, à la fin du XXe siècle, que la préséance du civil sur le religieux n'est pas respectée, sauf par les chrétiens. Jusqu'à présent, le choix matrimonial est fonction, non de la religion, mais de réseaux anciens de parentés, d'alliances, de clientèle, d'amitiés et des règles matrimoniales des différents systèmes dont la complexité a été étudiée par les anthropologues. La plupart des hommes et des femmes de toutes conditions respectent les choix familiaux. Ceux qui souhaitent les infléchir se référent au droit musulman. Les femmes qui l'étudient y ont recours pour maintenir les hommes chef de famille, ce qu'ils revendiquent bien entendu, mais il s'agit surtout pour elles de les obliger à assurer les charges du ménage et à renoncer à l'adultère. Afin d'être égales en droits entre épouses, elles exigent qu'une valeur juridique soit accordée au mariage religieux, plus courant que celui à l'état civil. Il n'est pas rare qu'un homme polygame marié religieusement passe devant le maire avec une épouse qui, seule, pourra hériter de ses biens. Ces femmes considèrent également que le recours au droit musulman permet, mieux que la justice civile, de faire reconnaître leurs droits, en cas de conflit, par la pression sociale et la conciliation.

L'opposition à la réforme du code de la famille est un défi au plan juridique, dans un contexte où les droits individuels sont reconnus. Des citoyens exigent que l'Etat donne aux tribunaux les outils leur permettant d'interpréter les lois en tenant compte de leurs valeurs. Deux personnalités politiques, difficilement qualifiables d'islamistes, l'altermondialiste, ancienne ministre Aminata Traoré [Diarra Diop, 
2009] et le Docteur Mariko du parti Sadi, ont été dans ce sens. Tous deux expriment la nécessité de la concertation et du dialogue entre les milieux politiques et religieux pour parvenir à des changements qui ne peuvent être imposés. La démocratie implique la participation populaire aux décisions, d'autant que celles-ci concernent la famille.

Les démocrates, favorables à la réforme, considèrent que le Président de la République a fait preuve d'écoute face à ce mouvement populaire. La capacité de mobilisation des associations islamiques est telle qu'elles ont un réel poids politique et permettent aux acteurs sociaux, quel que soit leur religion, de se faire entendre dans la sphère publique. Aminata Traoré regrette que les mouvements paysan et altermondialiste, malgré l'ampleur de leurs mobilisations, n'ait pas réussi, quant à eux, à empêcher l'adoption et la promulgation de la loi permettant les OGM. On s'interroge, avec elle, sur des réformes considérées comme une avancée par les bailleurs occidentaux, mais dans lesquelles une part importante de la société malienne ne se reconnaît pas ! 


\section{REFERENCES BIBLIOGRAPHIQUES}

Amin S. et Houtart F. (dir.) 2002, Mondialisation des résistances. L'état des luttes, L'Harmattan, Paris, 388 p.

Amselle J.L. 1990, Logiques métisses. Anthropologie de l'identité en Afrique et ailleurs, Payot, Paris.

- (1996) «L'étranger dans le monde manding et en Grèce ancienne : quelques points de comparaison », Cahiers d'Études africaines, XXXVI (4), $144: 755-76$.

BA Konare A. (dir.) (2009 (2008)), Petit précis de remise à niveau sur l'histoire africaine à l'usage du président Sarkozy, préface d'Elikia M'Bokolo, postface Catherine Clément, La découverte/poche, Paris.

BAYARD JF. (1983), «La revanche des sociétés africaines », Politique Africaine, 11, p. 95-12.

- (2004), Le gouvernement du monde. Une critique politique de la globalisation, Fayard, Paris.

BEBEy F. (2008 (1992)), Le ministre et le griot, Sépia, Poche.

Bierschenk T., Chauveau J.P. et Olivier de SARdan J.P. (dir.) (2000), Courtiers en développement, Karthala, Paris.

BLUNDO G. (1995), «Les courtiers du développement en milieu rural sénégalais », Cahiers d'Études africaines, XXXV (1), 137, p. 73-99.

BRAUMAN R. (2009), Humanitaire, diplomatie et droits de l'homme, éditions du Cygne Préface T. Todorov.

Burgat F. (2002 (1995)), L'islamisme en face, Paris, La Découverte, Poche Essais

2005, L'islamisme à l'heure d'Al Qaida. Réislamisation, modernisations, radicalisation, Paris, La Découverte.

Canut C. eт Smith É. (2006), «Pactes, alliances et plaisanteries », Cahiers d'études africaines, 184, ( http://etudesafricaines.revues.org/index6198.html). Coulon C. (1983), Les musulmans et le pouvoir en Afrique noire, Karthala, Paris.

Dahou T. (2005), «L'espace public face aux apories des études africaines », Cahiers d'Etudes africaines, XLV, 2, 178, p.327-349. 
DOUYON D. (2006), «Le discours diplomatique et démagogique du cousin $\begin{array}{lllll}\text { plaisant au Mali », Cahiers d'études africaines, } & 184\end{array}$ (http://etudesafricaines.revues.org/index6238.html)

Dozon J.-P. (2008), L'Afrique à Dieu et à Diable. Etats, ethnies et religions, ellipses, Paris.

Georges S., SAbelli F. (1994), Crédits sans frontières, la religion séculière de la Banque Mondiale, Fayard, Paris.

HoutART F. (1998), «La société civile: enjeux des luttes sociales pour l'hégémonie » in Société civile : lieu des luttes sociales, CETRI-l'Harmattan, vol. 1, Louvain-la-Neuve, Paris, p. 5-19.

- (2001), «Une nouvelle frontière » in Société civile mondiale la montée en puissance, Courrier de la Planète, 63, UNESCO-Most, vol. III, p. 15-18. (en ligne : unesco.org/most)

JONCKers D. (1987), La société minyanka du Mali, Connaissances des Hommes, Paris, L'Harmattan.

-1990 «Hiérogamie et stratégies matrimoniales» in F. Héritier, La complexité de l'alliance, Paris, Archives contemporaines : 125-148.

- 1994 "Le mythe d'une tradition communautaire villageoise dans la région Malisud" in J.P. Jacob et Ph. Lavigne Delville, Les associations paysannes en Afrique. Organisation et dynamiques, APAD-KARTHALA-IUED, 121-134.

1995a, «Territorialité et politique des sociétés initiatiques (Bamana-Minyanka, Mali) in Vincent J.F., Verdier R., Dory D., dir., La construction religieuse du territoire, Paris, L’Harmattan, pp. 183-196.

1995b, «Stratégies alimentaires et développement en région cotonnière du Mali-Sud » in Devisch R., De Boeck F., Jonckers D., dir., Alimentations, traditions et développement en Afrique intertropicale, Paris, L'Harmattan, pp. 219-247.

1997, «Puissance, sacralité et violence des pouvoirs chez les Minyanka bamana du Mali », in de Surgy A., Ed., Religion et pratiques de puissance, Paris, L'Harmattan, pp. 55-80.

1998, «"Le temps de prier est venu" Islamisation et pluralité religieuse dans le sud du Mali », Journal des Africanistes, 68 (1-2), pp. 21-45.

2006, «Résistances aux stratégies musulmanes de la France en Afrique occidentale, en région soudano-voltaïque » in P.J. Luizard, dir., Le choc 
colonial et l'islam. Les politiques religieuses des puissances coloniales en terre d'islam, Paris, La Découverte, textes à l'appui/histoire contemporaine, pp. 283-299.

2009, «Associations islamiques et enjeux démocratiques au Mali. De l'affirmation identitaire à la contestation de l'État et des institutions internationales » in Caratini S. (dir) La question du pouvoir en Afrique du Nord et de l'Ouest. Affirmations identitaires et enjeux de pouvoir, tome 2, Collection L'Ouest saharien, Hors série n ${ }^{\circ}$ 10, L'Harmattan, Paris, p.99-130.

LAGREE J.C. (2004), « Société civile internationale, un concept à réévaluer », in Oulahhib L.S. (dir.), «Age d'Or, Réforme, Altermonde », Esprit critique, vol $6, \mathrm{n}^{\circ} 2$, p. 52-69.

Lebeau Y, Niane B., Piriou A. et de Saint Martin M. [dir.] (2003), Etats et acteurs émergents en Afrique, Karthala, Paris.

Miran M. (2006), Islam, histoire et modernité en Côte d'Ivoire, Karthala, Paris.

Olivier DE SARDAN J.P (1997), Anthropologie et développement. Essai en socio-anthropologie du changement social, Karthala, Paris.

Ould Cheikh AbDel Wedoud (2004), «Espace confrérique, espace étatique: le mouridisme, le confrérisme et la frontière mauritanosénégalaise », in Marfaing L., Wippel S., Eds, Les relations transsahariennes à l'époque contemporaine. Un espace en constante mutation, Paris, KarthalaZMO, pp. 195-230.

Robinson D. (2004 (trad., 2000)), Sociétés musulmanes et pouvoir colonial français au Sénégal et en Mauritanie 1880-1920. Parcours d'accommodation, Paris, Karthala, 410p..

SAMSON F. (2005), Les marabouts de l'islam politique. Le Dahiratoul Moustarchidina Wal Moustarchidaty un mouvement néo-confrérique sénégalais, Paris, Karthala, 379 p.

Saul M. and RoYer P. (2001), West African Challenge to Empire Culture and History in the Volta-Bani Anticolonial War, Western African Studies, Ohio University Press, Athens, Oxford, James Currey. 
SchMitZ, J. (2008), « Migrants ouest-africains vers 1'Europe: historicité et espaces moraux » in Schmitz, J. (dir.) Migrants ouest-africains : miséreux, aventuriers ou notables ?, Politique africaine, ${ }^{\circ} 109$, p.5-15.

SCHULZ Dorothea E. (2003), "Political Factions, Ideological Fictions : the Controversy over Family Law Reform in Democratic Mali”, Islamic Law and Society, volume X, Leiden, Brill, pp. 132-164.

- (2006), "Promises of (Im)mediate Salvation : Islam Broadcast Media and the Remaking of Religious Experience in Mali", American Ethnologist, volume $33, \mathrm{n}^{\circ} 2$, pp. 210-229.

Seck A, 2008, La question musulmane au Sénégal (entre fin 80 et milieu 2000), thèse de doctorat Anthropologie, Université de Provence AixMarseille, $381 \mathrm{p}$.

SoARES B. (2004), «Islam and Public Piety in Mali » in Salvatore A. and Eickelman D. F. (ed.), Brill, Public Islam and the Common Good, Leiden, p. 205-226.

-(1996) "The Prayer Economy in a Malian Town", Cahiers d'Etudes africaines 144, XXVI, 4, pp. 739-753.

TAMARI T. (2010), «Des femmes savantes au Mali : XXe et XXIe siècles », communication Congrès des Etudes africaines en France, CEAN/IEP de Bordeaux 6-8 septembre 2010.

Traore A. (1999), L'étau, Actes Sud, Paris.

- (2002), Le viol de l'imaginaire, Fayard, Paris.

Ziegler J. (2002), Les nouveaux maîtres du monde. Et ceux qui leur résistent, Paris, Fayard.

-(2005), L'empire de la honte, Paris, Fayard.

\section{PRESSE ARCHIVES INFORMATIQUES}

DiARRA Diop M., (2009), Interview exclusive d'Aminata Traoré : «Le code de la famille est un défi à la démocratie!», $6 \mathrm{p}$, aminatatraorejournalduMali.com

KeITA S, (2009), « La contribution des Maliens de l'Extérieur au développement de leur pays », communication à la Conférence des Nations 
Unies sur le Commerce et le Développement (CNUCED), 29 juillet, Genève. keitaconferencecnucedbamakojuillet2009

Service des droits des femmes et de l'égalité, (2007), Synthèse de l'actualité 27 novembre, http://www.travail-solidarite.gouv.fr/ declarationdesegou

Forum des peuples : http://www.forumdespeuples.org/ 